\title{
Uplink Channel Estimation for Multi-user OFDM-based Systems
}

\author{
Carlos Ribeiro • M. Julia Fernández-Getino Garcia • Víctor P. Gil Jiménez • \\ Atílio Gameiro • Ana García Armada
}

\begin{abstract}
In this paper we propose a simple, yet fl xible and efficient channel estimator for the uplink in broadband orthogonal frequency division multiplexing (OFDM) systems. The processing is performed in the time-domain, by extracting the Channel's Impulse Response (CIR) for each user from a joint training signal. In this OFDM system, the pilot sequence we advocate, where all users share the same pilot sub-carriers, consists of one OFDM-symbol endowed with time-shifted properties per user, which isolates each user's CIR and is robust against multi-user interference. The feasibility of our approach is substantiated by system simulation results obtained using BRAN-A broadband mobile wireless channel model.
\end{abstract}

Keywords Channel estimation · OFDM $\cdot$ Multi-user $\cdot$ Uplink

\section{Introduction}

Orthogonal Frequency Division Multiplexing (OFDM) is the choice for a variety of wideband applications due to its robustness against frequency selective channels [1]. In addition, in a multi-user scenario, there exists the so-called multi-user diversity [2]: the probability that, at least, one user experiences good channel propagation conditions increases with the number

\section{Ribeiro}

Instituto Politécnico de Leiria, Morro do Lena, Alto Vieiro, 2411-901 Leiria, Portugal

e-mail: cribeiro@estg.ipleiria.pt

C. Ribeiro - A. Gameiro

Instituto de Telecomunicações, Universidade de Aveiro, Campo Universitário, 3810-193 Aveiro, Portugal e-mail: amg@av.it.pt

M. J. Fernández-Getino Garcia $(\varangle)$ · V. P. Gil Jiménez · A. García Armada University Carlos III de Madrid, Av. de la Universidad, 30-28911 Leganés, Madrid, Spain e-mail:mjulia@tsc.uc3m.es

V. P. G. Jimenez

e-mail: vgil@tsc.uc3m.es

A. García Armada

e-mail: agarcia@tsc.uc3m.es 
of users. If there are many users, it is highly probable that not all of them will experience poor channel propagation and at least one of them will have a good channel. Thus nowadays such multi-user schemes are rapidly growing to improve system efficien y.

Most of the systems require accurate channel estimation either for demodulation/decoding or resource allocation. Usually channel estimation methods are pilot-based, i.e. the channel information is extracted from known transmitted symbols that, although decreasing system efficien y since no data information is conveyed, they provide better performance than blind methods. In centralized networks, this problem is particularly important in the uplink, where multiple users must send pilots efficientl to the access point, so that it can perform the multiuser channel estimation accurately. In order to do so, in [3] overlapped pilots are proposed for channel estimation where different terminals utilize the same pilot sub-carriers avoiding the decrease in eff ciency as the number of users increases. However, the performance results are not very favorable.

In this paper, we present an uplink channel estimation method specifically adapted for wideband OFDM-based transmission systems, where several users share the same pilot positions with-in the frame, with minimal interference among them, but attaining better performance. The system must have dedicated sub-carriers for the transmission of those pilots. Perfect synchronization is assumed at the Base Station and the channel response is considered constant during each OFDM symbol.

\subsection{Notation}

Through the paper, the following notation is used. Bold and Capital Bold letters for vectors and matrices in time-domain and frequency-domain respectively. $E\{\cdot\}$ and [.] denote expectation and index respectively.

The remaining of the paper is organized as follows. Section 2 briefl presents the system model for multi-user OFDM-based systems. The multi-user channel estimation algorithm for the uplink scenario based on a time-domain procedure is developed in Sect. 3, and also, pilot sequences design with special shifting properties is presented. Simulation results and discussions are provided in Sect. 4. Finally, conclusions are drawn in Sect. 5.

\section{System Model for Multi-user OFDM in the Uplink}

Let us consider OFDM modulation over $N_{c}$ sub-carriers for transmission over a multipath fading channel. In the single-user case, the transmitted signal in frequency-domain is described by $\mathbf{S}=\mathbf{D}+\mathbf{P}$, where $\mathbf{S}$ is the column $N_{c}$-vector whose components $S[k]$, $k=0, \ldots, N_{c}-1$, are complex symbols transmitted at $k$ th sub-carrier. These complex symbols will belong to a two-dimensional constellation ( $M$-ary PSK or QAM). This complex symbol may be either a pilot $P[k]$ or data $D[k]$. The column $N_{c}$-vector $\mathbf{D}$ collects the data symbols, in which its $k$ th element $D[k]$ is data symbol at $k$ th sub-carrier. Similarly, the column $N_{c}$-vector $\mathbf{P}$ collects the pilot symbols, in which its $k$ th element $P[k]$ is a pilot value at $k$ th sub-carrier. Both vectors contain values at disjoint sub-carriers, and therefore, $S[k]=D[k]+P[k]$. If we defin the IDFT (Inverse Discrete Fourier Transform) $N_{c} \times N_{c}$ matrix,

$$
\mathbf{Q} \triangleq \frac{1}{N_{c}}\left(e^{j 2 \pi k n / N}\right)_{k, n=0,0}^{N_{c}-1, N_{c}-1}
$$


then the transmitted time-domain signal vector $\mathbf{s}$, whose components are $s[n], n=0, \ldots$, $N_{c}-1$, can be expressed with the IDFT transform pair as

$$
\mathbf{s}=\mathbf{Q S}=\mathbf{Q}(\mathbf{D}+\mathbf{P})=\mathbf{Q D}+\mathbf{Q P}=\mathbf{d}+\mathbf{p}
$$

where the time-domain transmitted data column $N_{c}$-vector $\mathbf{d}$ collects $d[n]$ components while pilot column $N_{c}$-vector $\mathbf{p}$ collects $p[n]$ components, yielding $s[n]=d[n]+p[n]$. The received signal in time-domain $r[n]$ will be given by $r[n]=s[n] * h[n]+w[n]$, where $h[n]$ is the Channel's Impulse Response (CIR) and $w[n]$ is the Additive White Gaussian Noise (AWGN). The channel's frequency response can be obtained via DFT transform and then $\mathbf{H}=\mathbf{Q h}$, where $\mathbf{h}$ and $\mathbf{H}$ are time-domain and frequency-domain channel column $N_{c^{-}}$ vectors, respectively. Each component of the frequency-domain vector $H[k], k=0, \ldots$, $N_{c}-1$ is the channel's frequency response at $k$ th sub-carrier.

For the OFDMA (Orthogonal Frequency Division Multiple Access) uplink, $U$ users simultaneously access the Base Station. All users perform OFDM modulation over $N_{c}$ subcarriers. In this case, the pilot values in frequency domain per $u$ th user, $u=0, \ldots, U-1$, will be denoted as $P_{u}[k]$, while the pilot sequence in time domain per $u$-th user is denoted as $p_{u}[n]$. The time-domain received signal in this multi-user scenario is then given by

$$
r[n]=\sum_{u=0}^{U-1} r_{u}[n]+w[n]=\sum_{u=0}^{U-1} p_{u}[n] * h_{u}[n]+w[n]
$$

where $r_{u}[n]$ is the received signal from $u$-th user and $h_{u}[n]$ is the channel impulse response for that user.

\section{Channel Estimation Method}

For the single-user case, Minn et al. [4] proposed the following pilot information to carry out their Most Significant Taps channel estimation method. Let the pilot symbols $P[k]$ be given by

$$
P[k]=\sum_{m=0}^{N_{c} / N_{f}-1} \delta\left[k-m N_{f}\right], 0 \leq k \leq N_{c}-1
$$

where $N_{f}$ is the spacing between pilot sub-carriers in frequency and $N_{c}$ is the total number of sub-carriers. If $N_{t}=\frac{N_{c}}{N_{f}}$ is integer, then the time-domain pilot signal is,

$$
\begin{aligned}
p[n] & =\frac{1}{N_{c}} \sum_{k=0}^{N_{c}-1} \sum_{m=0}^{N_{t}-1} \delta\left[k-m N_{f}\right] \exp \left(j 2 \pi \frac{k n}{N_{c}}\right) \\
& =\frac{1}{N_{c}}\left[e^{j 2 \pi \frac{0 n}{N_{c}}}+e^{j 2 \pi \frac{N_{f} n}{N_{c}}}+\cdots+e^{j 2 \pi \frac{\left(N_{c}-N_{f}\right) n}{N_{c}}}\right] \\
& = \begin{cases}\frac{1}{N_{f}}, & n=\ell \frac{N_{c}}{N_{f}}=\ell N_{t}, \quad \ell \in \mathbb{N}_{0} \\
0, & \text { others }\end{cases} \\
& =\frac{1}{N_{f}} \sum_{m=0}^{N_{f}-1} \delta\left[n-m N_{t}\right]
\end{aligned}
$$




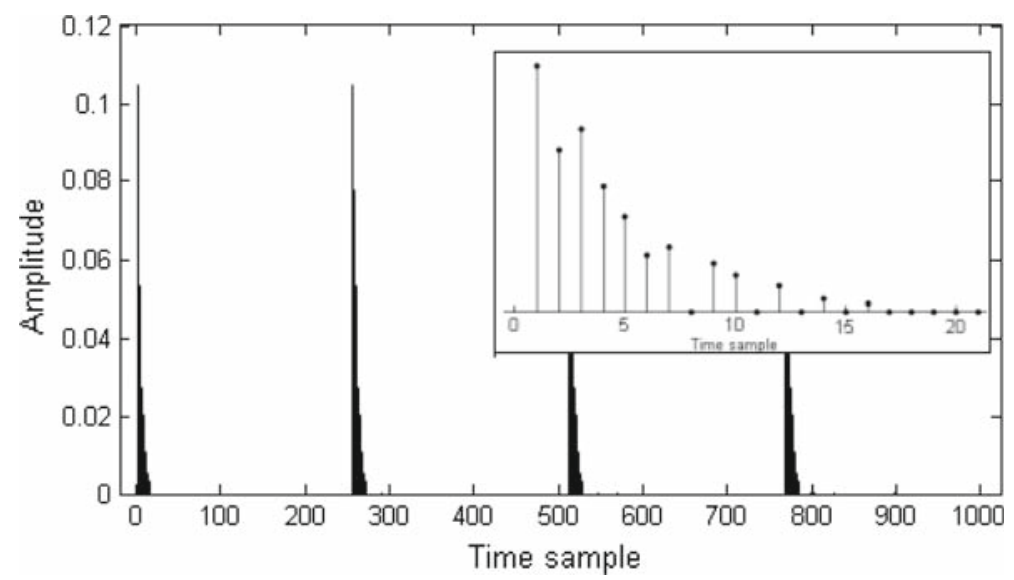

Fig. 1 Received signal, for the single-user case, when only pilot sub-carriers are sent, without loading data in the remaining ones. $N_{c}=1,024$

Equation (5) brings in evidence that the pilot vector in time domain appears as $N_{f}$ discrete samples uniformly separated $N_{t}$ samples. Transmitting this pilot signal through a wireless multipath channel with a maximum delay spread shorter than $\Delta t N_{t}$, where $\Delta t$ is the sampling time, will result in a received vector $r[n]$ containing $N_{f}$ scaled replicas of the channel's impulse response $h[n]$, independently corrupted by AWGN noise $w[n]$, as it can be seen in Fig. 1.

$$
r[n]=p[n] * h[n]+w[n]=\frac{1}{N_{f}} \sum_{m=0}^{N_{f}-1} h\left[n-m N_{t}\right]+w[n]
$$

For the purpose of illustration, Fig. 1 shows an example of a received pilot vector transmitted through an 18 path BRAN-A [5] wireless channel with a maximum delay spread of $390 \mathrm{~ns}$, a pilot distance $N_{f}=4$ and a sampling time $\Delta t=25 \mathrm{~ns}$. The zoomed figur in the top right corner of Fig. 1 details the frst received replica of the CIR.

The channel estimator changes the received vector by keeping $L$ samples (Most Significant Taps) of each replica of the channel impulse response, starting at positions $m N_{t}+1$, $m=0, \ldots, N_{f}-1$ and zeroing the remaining symbol samples,

$$
r^{\prime}[n]= \begin{cases}r[n], & n=m N_{t}+i ; i=0, \ldots, L-1 \\ 0, & \text { others }\end{cases}
$$

then, a new signal $\bar{r}[n]$ can be define averaging the $N_{f}$ replicas of the previous vector $r^{\prime}[n]$, padded with zeros up to $N_{c}$,

$$
\bar{r}[n]=\left\{\begin{array}{l}
\frac{1}{N_{f}} \sum_{m=0}^{N_{f}-1} r\left[m N_{t}+n\right], \quad n=1, \ldots, L \\
0, \quad n=L+1, \ldots, N_{c}
\end{array}\right.
$$


The estimate of the channel's frequency response at $k$-th sub-carrier $\hat{H}[k]$ is the result of the DFT of the signal $\bar{r}[n]$,

$$
\hat{H}[k]=D F T_{N_{c}}\{\bar{r}[n]\}
$$

where we defin this DFT $N_{c} \times N_{c}$ matrix as

$$
\mathbf{Q}^{\prime} \triangleq\left(e^{-j 2 \pi k n / N}\right)_{k, n=0,0}^{N_{c}-1, N_{c}-1}
$$

Most practical multipath channels will have its energy concentrated in a few significant taps, selecting the most significant ones of each replica and neglecting the remaining ones will result in a performance improvement [4] in comparison with DFT-based channel estimation methods [6]. The transmission of randomized data along with pilots will result in some performance degradation of the channel estimation as the data part of the kept samples of the received symbols will act as noise in the estimation process [4].

To deal with several users on the uplink, we propose to use a similar set-up, with all users using the same uncoded pilot sub-carriers in the same symbols that completely add up at the receiver, in the frequency domain. To be able to get estimates of each channel, each user will use different values for pilots. We propose a multi-user pilot sequence $P_{u}[k]$ in the frequency domain given by,

$$
P_{u}[k]=\sum_{m=0}^{N_{t}-1} \delta\left[k-m N_{f}\right] \exp \left(-j \frac{2 \pi}{U} u m\right) .
$$

As an example, for the case of $U=4$ users, the frequency-domain pilot vectors will be,

$$
\left\{\begin{array}{l}
\mathbf{p}_{0}=[+1,0 \ldots+1,0 \ldots+1,0 \ldots+1,0 \ldots+1 \ldots] \\
\mathbf{p}_{1}=[+1,0 \ldots-i, 0 \ldots-1,0 \ldots+i, 0 \ldots+1 \ldots] \\
\mathbf{p}_{2}=[+1,0 \ldots-1,0 \ldots+1,0 \ldots-1,0 \ldots+1 \ldots] \\
\mathbf{p}_{3}=[+1, \underbrace{0 \ldots}_{N_{f}-1},+i, 0 \ldots-1,0 \ldots+i, 0 \ldots+1 \ldots]
\end{array}\right.
$$

The use of these different pilot values for each user results in the shifting of the replicas of the received symbols for $u$-th user by $u \frac{N_{t}}{U}$ samples in the time-domain, thus allowing the Base Station to estimate the channel for each terminal, at the cost of some added interference from data of other terminals. The time domain pilot signal per $u$-th user $p_{u}[n]$ is then

$$
\begin{aligned}
p_{u}[n] & =\frac{1}{N_{c}} \sum_{k=0}^{N_{c}-1} \sum_{m=0}^{N_{t}-1} \delta\left[k-m N_{f}\right] e^{-j 2 \pi \frac{u}{U} m} e^{-j \frac{2 \pi}{N_{c}} k n} \\
& =\frac{1+\cdots+e^{-j 2 \pi \frac{u}{U}\left(N_{t}-1\right)} e^{-j \frac{2 \pi}{N_{c}}\left(N_{c}-N_{f}\right) n}}{N_{c}} \\
& = \begin{cases}\frac{1}{N_{f}}, & n=\frac{u}{U} N_{t}+m N_{t}, \quad m=0, \ldots, N_{f}-1 \\
0, & \text { others }\end{cases} \\
& =\frac{1}{N_{f}} \sum_{m=0}^{N_{f}-1} \delta\left[n-\frac{u}{U} N_{t}-m N_{t}\right], \frac{N_{t}}{U} \text { integer }
\end{aligned}
$$


or equivalently,

$$
\left\{\begin{array}{l}
p_{o}[n]=\frac{1}{N_{f}} \sum_{m=0}^{N_{f}-1} \delta\left[n-m N_{t}\right] \\
p_{1}[n]=\frac{1}{N_{f}} \sum_{m=0}^{N_{f}-1} \delta\left[n-\frac{1}{U} N_{t}-m N_{t}\right] \\
\vdots \\
p_{U-1}[n]=\frac{1}{N_{f}} \sum_{m=0}^{N_{f}-1} \delta\left[n-\frac{U-1}{U} N_{t}-m N_{t}\right]
\end{array}\right.
$$

Considering the transmission of symbols from $U$ users with independent channels and perfectly synchronized, the received signal, recalling (3), will be,

$$
\begin{aligned}
r[n]= & \sum_{u=0}^{U-1} p_{u}[n] * h_{u}[n]+w[n] \\
= & \sum_{u=0}^{U-1} \frac{1}{N_{f}} \sum_{m=0}^{N_{f}-1} \delta\left[n-\frac{u}{U} N_{t}-m N_{t}\right] * h_{u}[n]+w[n] \\
& \sum_{m=0}^{N_{f}-1} h_{0}\left[n-m N_{t}\right] \\
& \frac{\sum_{m=0}^{N_{f}-1} h_{1}\left[n-\frac{1}{U} N_{t}-m N_{t}\right]}{N_{f}}+\frac{\sum_{m=0}^{N_{f}-1} h_{U-1}\left[n-\frac{U-1}{U} N_{t}-m N_{t}\right]}{N_{f}}+w[n] .
\end{aligned}
$$

As an example, Fig. 2 shows a realization where one OFDM-symbol carrying only pilots (data sub-carriers are not loaded in this example) from each user is transmitted through independent BRAN-A channels and arrives synchronized at the Base Station.

The Base Station channel estimator changes the vector of the received samples by keeping $L$ samples of each replica starting at positions $m N_{t} \frac{1}{U}+1, m=0, \ldots, N_{f} U-1$ and zeroing the remaining vector samples. We defin signal $\bar{r}_{u}[n]$ per $u$-th user, each made up of the averaging of the $N_{f}$ replicas pertaining to the desired user, padded with zeros up to $N_{c}$,

$$
\bar{r}_{u}[n]=\left\{\begin{array}{l}
\frac{1}{N_{f}} \sum_{m=0}^{N_{f}-1} r\left[m N_{t}+N_{t} u / U+n\right], \quad n=1, \ldots, L \\
0, \quad n=L+1, \ldots, N_{c}
\end{array}\right.
$$

The estimates of the channel's frequency response at $k$-th sub-carrier for $u$-th user $\hat{H}_{u}[k]$ is the result of the DFT of the signal $\bar{r}_{u}[n]$, yielding $\hat{H}_{u}[k]=D F T_{N_{c}}\left\{\bar{r}_{u}[n]\right\}$. Observing Fig. 2 it is clear that the maximum number of elements that can use the same pilot carriers is limited and depends on the pilot distance $N_{f}$ and the channel's maximum delay spread $\tau_{u, \max }$ for the $u$-th user:

$$
U \leq \frac{N_{c} \Delta t}{N_{f} \tau_{u, \max }} .
$$

The overall system uses $U$ times less pilot carriers, improving the performance in terms of system's power eff ciency. 

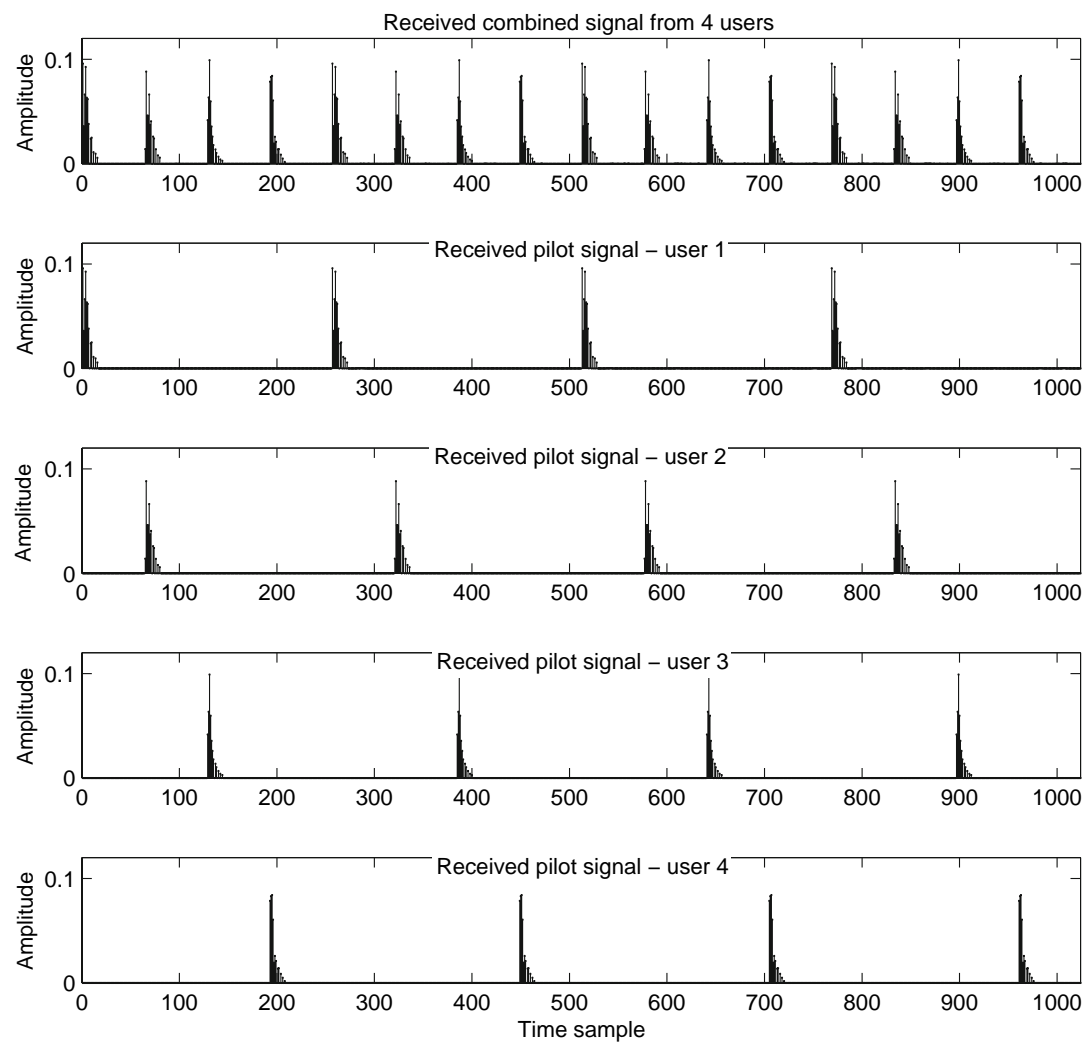

Fig. 2 Received signal for the multi-user case $(U=4)$, when only pilot sub-carriers are sent (data sub-carriers are not loaded)

In the presence of a time-variant channel, the channel estimation in the time direction may be performed using some sort of simple interpolator (quadratic, cubic, ...) to keep the overall complexity low. In this case, a 2-D diamond shaped pilot pattern should be used [7] to achieve the best performance with the same complexity.

\section{Performance Evaluation}

A simulation scenario with $U=4$ users was implemented, where all users share the same pilot sub-carriers. Since this channel estimation method performs frequency-domain estimation, the measurements presented in this section are only with respect to the symbols that carry both pilots and data. Channel estimation in the time direction, that would involve estimating the channel's frequency response of the symbols carrying only data, is not considered. The system uses $N_{c}=1,024$ QPSK modulated sub-carriers, with a pilot separation of $N_{f}=4$ carriers and a sampling time $\Delta t=25 \mathrm{~ns}$. Figure 3 shows the comparison between real and estimated channel's frequency response, when the four users transmit symbols through independent BRAN-A channels. The receiver's signal to noise ratio was set to $S N R=10 \mathrm{~dB}$ and $L=10$ samples from each replica were utilized. 

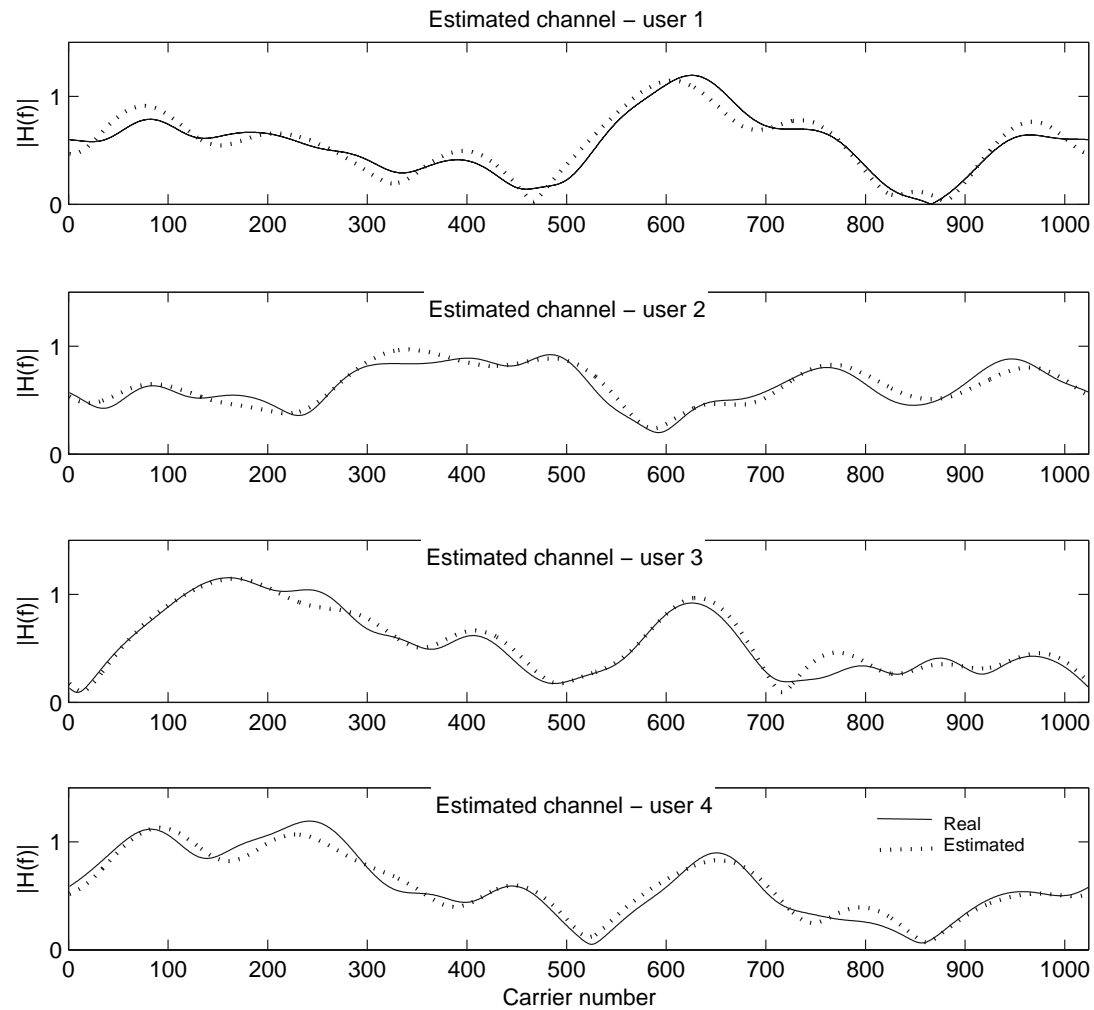

Fig. 3 Comparison between real and estimated channel's frequency response for the four users. The solid line refers to the real channel while dashed line represents the estimated channel

To assess the performance of the proposed method, several simulations were performed for different SNR values. Figure 4 presents several performance curves of Mean Squared Error (MSE) for channel estimation, averaged over the four users, for different values of $L$, when the $S N R$ at the input of the receiver ranges from 0 to $30 \mathrm{~dB}$. Each user transmitted symbols (carrying pilots and data) through independent BRAN-A channels. For comparison purposes an LS-DFT [6] channel estimation scheme was also implemented in order to give a fair comparison. Each user's pilots where multiplexed in the frequency domain in disjoint sub-carriers, so that the Base Station could estimate the $U$ different channels. In this scenario, each user used $U$ times less pilots, resulting in the same overall pilot density for both methods. The attained channel estimation MSE (averaged over the $U$ users) is also included in Fig. 4.

The utilization of the proposed method implies a trade-off between the amount of distortion introduced by removing energy from significant channel taps and the amount of noise inputed to the estimation process. A MSE noise floo is present due to removing some of the channel's energy and it will be lower as the number of samples $L$ is increased (at the price of a worse performance for low values of SNR.) In the region were the channel distortion is not the dominant factor on the performance (low SNR values), this method outperforms LS-DFT channel estimation considerably.

The dependence of the presented method on the pilot distance $N_{f}$ is an important topic to address to complete its evaluation. Figure 5 depicts several curves of the attained channel 


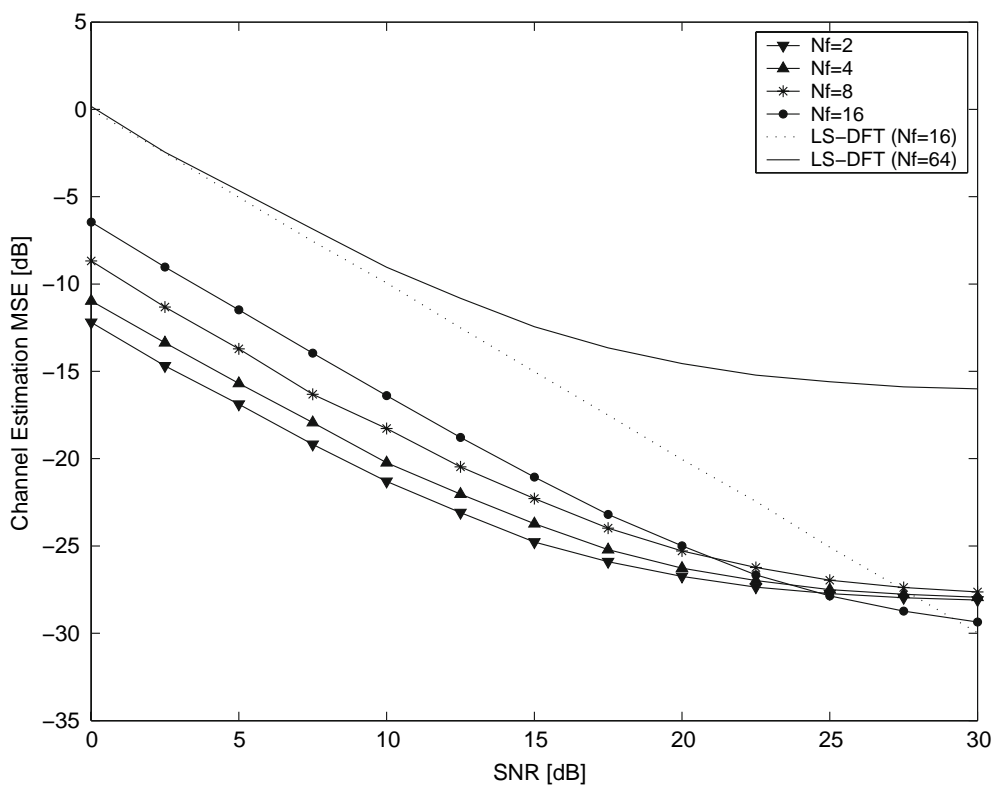

Fig. 4 MSE performance as a function of $L$. The dashed line refers to DFT-based channel estimation method for one user

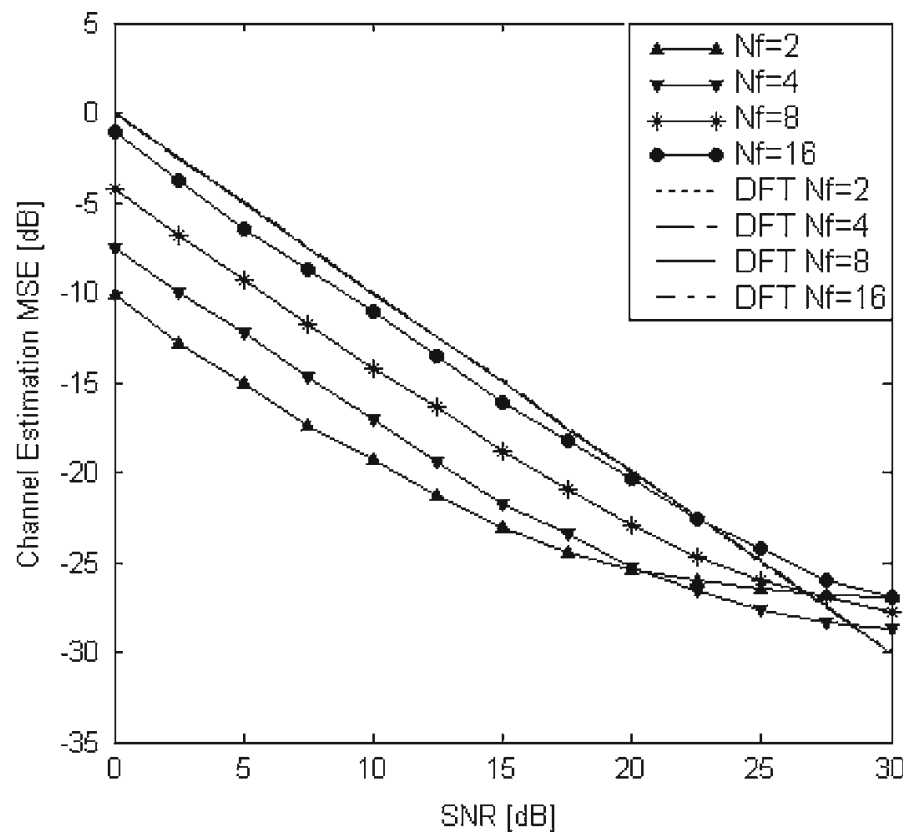

Fig. 5 Performance dependence on the pilot distance $N_{f}$ 
estimation MSE for different values of SNR and $N_{f}$ when $L=12$ samples were utilized. The LS-DFT channel estimation performance for $N_{f}$ values of 16 and 64 is also included in the f gure. The performance of the reference method is mainly independent on the pilot distance $N_{f}$ as along as the Nyquist Criterion is fulfilled

The line corresponding to the LS-DFT method with $N_{f}=64$ depicts a situation were the Nyquist Criterion is not fulfilled The aliasing causes severe degradation on the channel estimate for higher values of SNR. In the investigated method, in the equivalent situation $\left(N_{f}=16\right)$, the channel estimator still performs well, achieving a low MSE for the range of simulated SNRs.

Observing (15), the received signal $r_{u}[n]$ of user $u$ can be written as:

$$
\begin{aligned}
r_{u}[n] & =\frac{1}{N_{f}} \sum_{m=0}^{N_{f}-1} \delta\left[n-\frac{u}{U} N_{t}-m N_{t}\right] * h_{u}[n] \\
& =\frac{1}{N_{f}} \sum_{m=0}^{N_{f}-1} h_{u}\left[n-\frac{u}{U} N_{t}-m N_{t}\right]
\end{aligned}
$$

and signal power $S_{r_{u}}$ is,

$$
\begin{aligned}
S_{r_{u}} & =E\left\{\left|r_{u}[n]\right|^{2}\right\} \\
& =E\left\{\left|\frac{1}{N_{f}} \sum_{m=0}^{N_{f}-1} h_{u}\left[n-\frac{u}{U} N_{t}-m N_{t}\right]\right|^{2}\right\}=\frac{1}{N_{f}} S_{H_{u}}
\end{aligned}
$$

where $S_{H_{u}}$ is the channel power for user $u$. The power of each of the $U$ vectors $\bar{r}_{u}[n]$ define in (16) is

$$
S_{\bar{r}_{u}}=\frac{1}{N_{f}} S_{r_{u}}=\frac{1}{N_{f}^{2}} S_{H_{u}}
$$

Considering that the noise samples that make up $w[n]$ in (16) are independent and identically distributed with a variance $\sigma_{N}^{2}$, then, the noise term in (16), after averaging the $N_{f}$ replicas will be $\sigma_{N, \text { avg }}^{2}=\frac{\sigma_{N}^{2}}{N_{f}}$ resulting in a signal to noise ratio before the DFT of

$$
S N R_{\text {ChEst }}=\frac{S_{\bar{r}_{u}}}{\sigma_{N, a v g}^{2}}=\frac{1}{N_{f}} \frac{S_{H_{u}}}{\sigma_{N}^{2}} .
$$

Equation (21) justifie the performance presented in Fig. 5. In the region where noise is the dominant factor on the performance (low SNR values), decreasing the pilot distance will improve the channel estimation. This effect grows smaller as the received SNR increases and the channel distortion becomes increasingly more important.

\section{Conclusions}

We have proposed a simple channel estimator for multi-user OFDM-based systems in the uplink scenario. It works in the time-domain, by averaging each user's CIR replicas, which appear separated due to the use of the proposed pilot sequences with time-shifting properties. Our estimator can be easily used in either acquisition (preamble-based) or tracking (pilottones based) mode, and its structure remains the same for any type of training pattern in 
the two-dimensional time-frequency space. We have also provided the pilot sequences that allows identificatio of the multi-user channel by ensuring special time-shifting properties. The feasibility of our approach was substantiated by computer simulation results obtained for BRAN-A broadband wireless channel models.

Acknowledgements This work has been partly funded by the Spanish government with projects MACAWI (TEC 2005-07477-c02-02), MAMBO2 (CCG06-UC3M-TIC-0698), European project CRUISE (IST-2004027738), Spanish-Portuguese Integrated Action (HP2006-0025) and European COST Action 289 and is a result of work done within this European action.

\section{References}

1. Nee, R. V., \& Prasad, R. (2000). OFDM For wireless multimedia communications. 1st ed. Artech House.

2. Larsson, E. G. (2004). On the combination of spatial diversity and multiuser diversity. IEEE Comunications Letters, 8(8), 517-519.

3. Sternad, M., \& Aronsson, D. (2005). Channel estimation and prediction for adaptive OFDMA/TDMA uplinks, based on overlapping pilots. In Proceedings of the IEEE International Conference on Acoustics, Speech, and Signal Processing (ICASSP), Vol. 3, March 2005.

4. Minn, H., \& Bhargava, V. (2000). An investigation into time-domain approach for OFDM channel estimation. IEEE Transactions on Broadcasting, 46(4), 240-248.

5. Medbo, J. (1998). Channel Models for Hiperlan/2 in different indoor scenarios. ETSI BRAN doc. 3ERI085b.

6. Edfors, O., Sandell, M., van de Beek, J.-J., Wilson, S.K., \& Borjesson, P. O. (1996). Analysis of DFT-based channel estimators for OFDM. Research Report TULEA 1996:17, Division of Signal Processing, Lulea University of Technology, Sweden.

7. Choi, Ji-Woong, \& Lee, Yong-Hwan (2005). Optimum pilot pattern for channel estimation in OFDM systems. IEEE Transactions on Wireless Communications, 3(5), 2083-2088.

\section{Author Biographies}

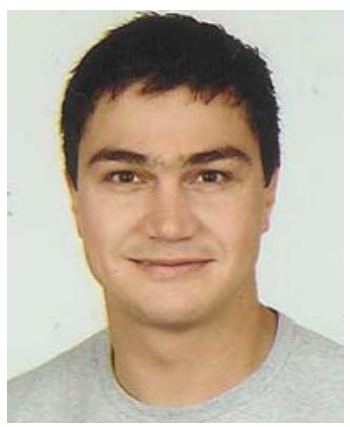

Carlos Ribeiro received his Licenciatura (5 year course) and his MSc in Electronic Engineering from the University of Coimbra in 1996 and 2003 respectively. He is currently an Assistant Lecturer in the Polytechnic Institute of Leiria, and a researcher at the Instituto de Telecomunicações-Pólo de Aveiro. His research interests lie in signal processing for multicarrier communication systems. He is currently working in his $\mathrm{PhD}$ thesis in iterative schemes for joint detection and estimation for MC-CDMA systems.

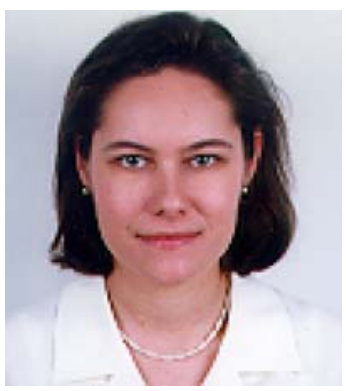

M. Julia Fernández-Getino García is working as Associate Profesor at the University Carlos III de Madrid, Spain. Her research interests include multi-carrier communications and signal processing for wireless systems. She visited Bell Laboratories, Lund University and Politecnico di Torino. She received the best "Master Thesis" award and the thesis award from the Professional Association of Telecommunication Engineers of Spain, and the "Student Paper Award" at the IEEE 10th International Symposium PIMRC. 

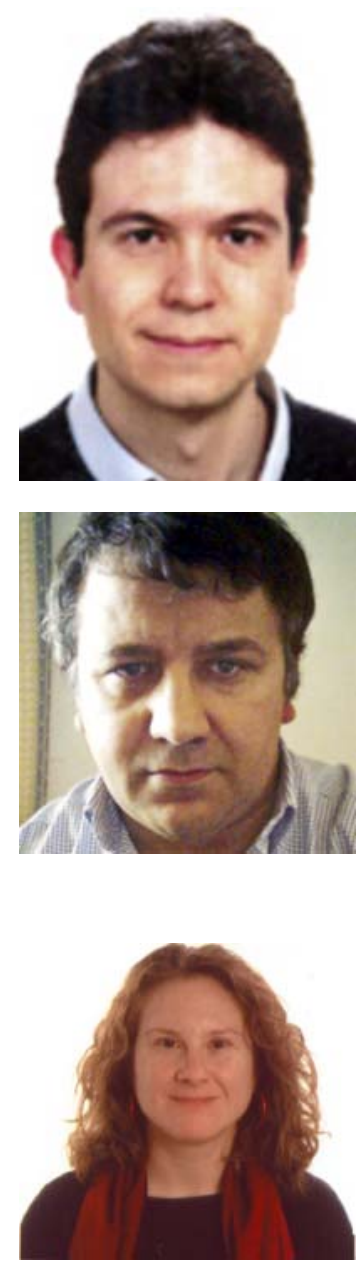

Víctor P. Gil Jiménez is with the Department of Signal Theory and Communications at the University Carlos III de Madrid as an Assistant Professor. He worked at the Spanish Antarctica Base in 1999 as Communications Staff. He visited University of Leeds and Chalmers Technical University in 2003 and 2004 respectively. His research interests include multicarrier communications and signal processing for wireless systems.

Atílio Gamiro received his Licenciatura (5 years) and his Ph.D from the University of Aveiro, 1985 and 1993 respectively. He is currently a Professor in the Department of Electronics and Telecommunications of the University of Aveiro, and a researcher at the Instituto de Telecomunicações, where he is head of group. His main interests lie in signal processing for digital communications and communication protocols. His current research activities involve space-time-frequency algorithms for the broadband component of $4 \mathrm{G}$ systems and joint design of layers 1 and 2.

Ana García Armada is currently working as an Associate Professor at the University Carlos III de Madrid, Spain where she has occupied several management positions. She has participated in various national and international research projects and has published 17 papers in international journals. She has performed research stays in ESA-ESTEC, Kansas University, Stanford University and Bell Labs. Her research interests are multicarrier and MIMO techniques. 